\title{
TLR 2/6/9 Agonist PUL-042
}

National Cancer Institute

\section{Source}

National Cancer Institute. TLR 2/6/9 Agonist PUL-042. NCI Thesaurus. Code C135091.

A solution consisting of a combination of two toll-like receptor (TLR) ligands: Pam2CSK4 acetate (Pam2), a synthetic diacylated lipopeptide (LP) that is an agonist of TLR2 and TLR6 (T LR2/6) and the TLR9 agonist oligodeoxynucleotide (ODN) M362, which contains unmethylated CpG-based dinucleotides, with potential immunostimulating activity. Upon administration via inhalation of the PUL-042 solution, the components in PUL-042 bind to and activate TLRs on lung epithelial cells. This induces the epithelial cells to produce peptides and reactive oxygen species (ROS) against pathogens, including bacteria, fungi and viruses, that are present in the lungs. M362, through binding of the $\mathrm{CpG}$ motifs to TLR9 and subsequent TLR9-mediated signaling, initiates the innate immune system and activates macrophages, natural killer (NK) cells, B-cells, and plasmacytoid dendritic cells (pDCs), stimulates interferon-alpha (IFN-a) production and induces a T-helper 1 cells (Th1)-mediated immune response. Pam2CSK4, through TLR2/6, activates the production of T-helper 2 cells (Th2), leading to the production of Th2-specific cytokines. Altogether, this protects against and kills the pathogens present in the lungs and prevents pulmonary infection. TLRs play fundamental roles in both pathogen recognition and the activation of innate immunity. 